General Letters in Mathematics (GLM) 1 (1) (2016) 1-10
General Letters in Mathematics (GLM)
Science
Weflection

\title{
Modified Newton's Methods with Seventh or Eighth -Order Convergence
}

\author{
Husnia Mohamed Eldanfour \\ Faculty of Industrial Technology - Misurata -Libya -PO Box 996 Misurata -Libya. \\ hsniam@yahoo.com
}

\begin{abstract}
In this paper, we consider a modification of the Newton's method which produce iterative method with fourth-order of convergence have been proposed in [4] and obtain new methods with ( seventh or eighth )-order convergence for solving non-linear equations. A general error analysis providing the higher order of convergence is given. Per iteration the new methods require one additional evaluation of the function. Numerical examples are also included the performance of the new methods.
\end{abstract}

Indexing terms/Keywords

Newton's method, Order of convergence, Function evaluations.

\section{SUBJECT CLASSIFICATION}

MSC[2010]:. 65K05,65K99.

\section{Introduction}

Newton's method for the approximation of the root $\xi$ of a nonlinear equation (or system of non-linear equations) is well known, in paper [6] Newton's method may be seen as the approximation of the indefinite integral arising from Newton's theorem

$f(x)=f\left(x_{n}\right)+\int_{x_{n}}^{x} f^{\prime}(t) d t$

by using the rectangular rule (the Newton- Cotes quadrature formula of order zero) for the computation of the integral in (1).

$\int_{x_{n}}^{x} f^{\prime}(t) d t \cong\left(x-x_{n}\right) f^{\prime}\left(x_{n}\right)$

And, looking for $f(x)=0$, we obtain the new value

$x_{n+1}=x_{n}-\frac{f\left(x_{n}\right)}{f^{\prime}\left(x_{n}\right)}$

Some new modified Newton's methods with third-order convergence have been developed in $[1,2,3,6]$, by considering different quadrature formula for the computation of the indefinite integral of (1). 
Weerakoom-.Fernando [5] used the trapezoidal rule to compute the integral of (1) to obtain the implicit method

$x_{n+1}=x_{n}-2 \frac{f\left(x_{n}\right)}{f^{\prime}\left(x_{n+1}\right)+f^{\prime}\left(x_{n}\right)}$

and, replacing $f^{\prime}\left(x_{n+1}\right)$ with $f^{\prime}\left(x_{n+1}^{*}\right)$; where $x_{n+1}^{*}=x_{n}-f\left(x_{n}\right) / f^{\prime}\left(x_{n}\right)$ is the classical Newton's iterate, they obtained the explicit with third-order convergence:

$\mathrm{x}_{\mathrm{n}+1}=\mathrm{x}_{\mathrm{n}}-2 \frac{\mathrm{f}\left(\mathrm{x}_{\mathrm{n}}\right)}{\mathrm{f}^{\prime}\left(\mathrm{x}_{\mathrm{n}+1}^{*}\right)+\mathrm{f}^{\prime}\left(\mathrm{x}_{\mathrm{n}}\right)}$

$\mathrm{x}_{\mathrm{n}+1}^{*}=\mathrm{x}_{\mathrm{n}}-\frac{\mathrm{f}\left(\mathrm{x}_{\mathrm{n}}\right)}{\mathrm{f}^{\prime}\left(\mathrm{x}_{\mathrm{n}}\right)}$

The midpoint rule for the integral of (1) gives that [3]

$x_{n+1}=x_{n}-\frac{f\left(x_{n}\right)}{f^{\prime}\left(\left(x_{n}+x_{n+1}^{*}\right) / 2\right)}$

$\mathrm{x}_{\mathrm{n}+1}^{*}=\mathrm{x}_{\mathrm{n}}-\frac{\mathrm{f}\left(\mathrm{x}_{\mathrm{n}}\right)}{\mathrm{f}^{\prime}\left(\mathrm{x}_{\mathrm{n}}\right)}$

For any quadrature formula of order higher than zero to approximating the integral of (1), the following theorem gives that modified Newton's methods have third-order convergence [3].

Theorem 1.1 The modified Newton's methods obtained by approximating the integral by the quadrature formula of order at least one, and writing the explicit form the obtained implicit method by replacing $\mathrm{X}_{\mathrm{n}+1}$ with $\mathrm{x}_{\mathrm{n}+1}^{*}$ given by $x_{n+1}^{*}=x_{n}-\frac{f\left(x_{n}\right)}{f^{\prime}\left(x_{n}\right)}$

is, if $\xi$ is a simple root of $\mathrm{f}\left(\mathrm{x}_{\mathrm{n}}\right)$, of order three with

$$
g_{m}^{\prime \prime \prime}(\xi)=\frac{f^{\prime \prime \prime}(\xi)}{f^{\prime}(\xi)}\left[3 \sum_{i=1}^{m} A_{i} \tau_{i}^{2}-1\right]+\frac{3}{2}\left(\frac{f^{\prime \prime}(\xi)}{f^{\prime}(\xi)}\right)^{2}
$$

where $g_{m}\left(x_{n}\right)=x_{n}-\frac{f\left(x_{n}\right)}{\sum_{i=1}^{m} A_{i} f^{\prime}\left(\eta_{i}^{*}\right)}, \eta_{i}^{*}=x_{n}-\tau_{i}\left(x_{n+1}^{*}-x_{n}\right), \tau_{i}$ are the knots, in [0,1], and $\mathrm{A}_{\mathrm{i}}$ are the weight of the quadrature formula used.

But, in [1] instead of using the Newton's theorem for $y=f(x)$, Homeier uses it for the inverse function $x(y)$ $\mathrm{x}(\mathrm{y})=\mathrm{x}\left(\mathrm{y}_{\mathrm{n}}\right)+\int_{\mathrm{y}_{\mathrm{n}}}^{\mathrm{y}} \mathrm{x}^{\prime}(\mathrm{t}) \mathrm{dt}$

to obtain a class of cubically convergent type methods, the best efficient one of which is

$x_{n+1}=x_{n}-\frac{f\left(x_{n}\right)}{2}\left(\frac{1}{f^{\prime}\left(x_{n}\right)}+\frac{1}{f^{\prime}\left(x_{n+1}^{*}\right)}\right)$

And, in [2] they considered the approximation of the indefinite integral (1) on a new interval of integration arising from Newton's theorem

$$
f(x)=f\left(y_{n}\right)+\int_{y_{n}}^{x} f^{\prime}(t) d t .
$$

where $y_{n}=x_{n}+f\left(x_{n}\right) / f^{\prime}\left(x_{n}\right)$.

by using the mid-point rule to approximate the right integral of (6) 


$$
\int_{y_{n}}^{x} f^{\prime}(t) d t \cong\left(x-y_{n}\right) f^{\prime}\left(\frac{x+y_{n}}{2}\right)
$$

A general implicit method is then obtained by approximating the integral in (6) by the mid-point rule (7) and looking for $\mathrm{f}(\mathrm{x})=0$, so that we have

$x_{n+1}=y_{n}-\frac{f\left(y_{n}\right)}{f^{\prime}\left(\left(x_{n+1}+y_{n}\right) / 2\right)}$

we can obtain an explicit method by replacing $\mathrm{f}^{\prime}\left(\left(\mathrm{x}_{\mathrm{n}+1}+\mathrm{y}_{\mathrm{n}}\right) / 2\right)$ with $\mathrm{f}^{\prime}\left(\left(\mathrm{x}_{\mathrm{n}+1}^{*}+\mathrm{y}_{\mathrm{n}}\right) / 2\right)$; where $\mathrm{x}_{\mathrm{n}+1}^{*}=\mathrm{x}_{\mathrm{n}}-\mathrm{f}\left(\mathrm{x}_{\mathrm{n}}\right) / \mathrm{f}^{\prime}\left(\mathrm{x}_{\mathrm{n}}\right)$, they obtained the explicit with third-order convergence:

$x_{n+1}=x_{n}-\frac{f\left(x_{n}+f\left(x_{n}\right) / f^{\prime}\left(x_{n}\right)\right)-f\left(x_{n}\right)}{f^{\prime}\left(x_{n}\right)}$.

Theorem 1.2 Assume that the function $f: D \subset R \rightarrow R$ has a simple root $\xi \in D$, where $D$ is an open interval. If $f(x)$ has first, second and third derivatives in the interval D, then the methods defined by (3), (4), (5), and (8) converge three to $\xi$ in a neighborhood of $\xi$, and satisfy the following error equation:

$e_{n+1}=\omega e_{n}^{3}+O\left(e_{n}^{4}\right)$.

Where $\omega=C_{2}^{2}+\frac{1}{2} C_{3}$ for method (4), $\omega=C_{2}^{2}-\frac{1}{4} C_{3}$ for method (5), $\omega=C_{2}^{2}-C_{3}$ for method (6), and $\omega=2 C_{2}^{2}-2 C_{3}$ for method (9). [1,2,3,5]

Kung[4] developed a one-parameter family of fourth-order methods, which written as

$\mathrm{y}_{\mathrm{n}}=\mathrm{x}_{\mathrm{n}}-\frac{\mathrm{f}\left(\mathrm{x}_{\mathrm{n}}\right)}{\mathrm{f}^{\prime}\left(\mathrm{x}_{\mathrm{n}}\right)}$

$\mathrm{x}_{\mathrm{n}+1}=\mathrm{y}_{\mathrm{n}}-\left[\frac{\mathrm{f}\left(\mathrm{x}_{\mathrm{n}}\right)+\beta \mathrm{f}\left(\mathrm{y}_{\mathrm{n}}\right)}{\mathrm{f}\left(\mathrm{x}_{\mathrm{n}}\right)-(\beta-2) \mathrm{f}\left(\mathrm{y}_{\mathrm{n}}\right)}\right] \frac{\mathrm{f}\left(\mathrm{y}_{\mathrm{n}}\right)}{\mathrm{f}^{\prime}\left(\mathrm{x}_{\mathrm{n}}\right)}$.

where $\beta$ is a constant. In particular, the special method for $\beta=-1 / 2$ is as follows:

$\mathrm{y}_{\mathrm{n}}=\mathrm{x}_{\mathrm{n}}-\frac{\mathrm{f}\left(\mathrm{x}_{\mathrm{n}}\right)}{\mathrm{f}^{\prime}\left(\mathrm{x}_{\mathrm{n}}\right)}$

$x_{n+1}=y_{n}-\left[\frac{2 f\left(x_{n}\right)-f\left(y_{n}\right)}{2 f\left(x_{n}\right)-5 f\left(y_{n}\right)}\right] \frac{f\left(y_{n}\right)}{f^{\prime}\left(x_{n}\right)}$.

In this paper, we will present two new modifications of modified Newton's methods with fourth- order convergence by using Newton's theorem for the function $\mathrm{f}(\mathrm{x})$ on a new interval of integration, and obtain a higher order methods.

\section{A new modifications of Newton's method and analysis of convergence}

To derive the new method, we consider the computation of the indefinite integral on a new interval of integration arising from Newton's theorem

$f(x)=f\left(z_{n}\right)+\int_{z_{n+1}}^{x} f^{\prime}(t) d t$. 
by using the rectangular rule to approximate the right integral of (10) we have

$\mathrm{x}_{\mathrm{n}+1}=\mathrm{z}_{\mathrm{n}}-\frac{\mathrm{f}\left(\mathrm{z}_{\mathrm{n}}\right)}{\mathrm{f}^{\prime}\left(\mathrm{z}_{\mathrm{n}}\right)}$.

where $z_{n}$ is the above modification of Newton's method (9).

As we can see, this method consists of three evaluations of the function and two evaluations of the first derivative per iteration, which has efficiency index 1.5157. In order to obtain high efficiency index, we approximate $\mathrm{f}^{\prime}\left(\mathrm{z}_{\mathrm{n}}\right)$ using Lagrange's interpolation polynomial [5] as follows:

$$
\begin{aligned}
f^{\prime}\left(z_{n}\right)= & \frac{\left(z_{n}-y_{n}\right)}{\left(x_{n}-y_{n}\right)\left(x_{n}-z_{n}\right)} f\left(x_{n}\right)+\frac{\left(z_{n}-x_{n}\right)}{\left(y_{n}-x_{n}\right)\left(y_{n}-z_{n}\right)} f\left(y_{n}\right)+ \\
& \frac{\left(z_{n}-x_{n}\right)+\left(z_{n}-y_{n}\right)}{\left(z_{n}-x_{n}\right)\left(z_{n}-y_{n}\right)} f\left(z_{n}\right) \\
= & \frac{\left[\begin{array}{l}
\left(z_{n}-x_{n}\right)^{2} f\left(y_{n}\right)+\left(y_{n}-x_{n}\right)\left(y_{n}-z_{n}\right) f\left(z_{n}\right)- \\
\left(y_{n}-z_{n}\right)^{2} f\left(x_{n}\right)-\left(y_{n}-x_{n}\right)\left(z_{n}-x_{n}\right) f\left(z_{n}\right)
\end{array}\right]}{\left(y_{n}-x_{n}\right)\left(y_{n}-z_{n}\right)\left(z_{n}-x_{n}\right)}
\end{aligned}
$$

Theorem 2.1. Assume that the function $f: D \subset R \rightarrow R$ has a simple root $\xi \in \mathrm{D}$, where $D$ is an open interval. If $\mathrm{f}(\mathrm{x})$ has first, second and third derivatives in the interval $D$, and $\mathrm{z}_{\mathrm{n}}$ is defined by (9) satisfies

$$
\begin{aligned}
z_{n}-\xi= & -C_{2} C_{3} e_{n}^{4}+\left(-2 C_{2} C_{4}+2 C_{2}^{2} C_{3}+\frac{3}{2} C_{2}^{4}-2 C_{3}^{2}\right) e_{n}^{5}+ \\
& \left(-12 C_{2} C_{5}-7 C_{3} C_{4}+3 C_{2}^{2} C_{4}+6 C_{3} C_{2}^{3}-\frac{35}{4} C_{2}^{5}\right) e_{n}^{6}+O\left(e_{n}^{7}\right)
\end{aligned}
$$

and $e_{n}=x_{n}-\xi$, then the method defined by (9) and (11) are of eighth-order

$$
e_{n+1}=C_{2}^{3} C_{3} e_{n}^{8}+O\left(e_{n}^{9}\right) \text {. }
$$

while the method defined by (9), (11) and (12) is of seventh-order

$$
e_{n+1}=-C_{2}^{2} C_{3}^{2} e_{n}^{7}+O\left(e_{n}^{8}\right)
$$

Proof: let $\xi$ be a simple root of $f(x)$ (i.e., $\mathrm{f}(\xi)=0$ and $\left.\mathrm{f}^{\prime}(\xi) \neq 0\right)$ and $\mathrm{e}_{\mathrm{n}}=\mathrm{x}_{\mathrm{n}}-\xi$.

Using the Taylor's expansion, we have

$$
\begin{aligned}
\mathrm{f}\left(\mathrm{x}_{\mathrm{n}}\right)= & \mathrm{f}(\xi)+\mathrm{f}^{\prime}(\xi) \mathrm{e}_{\mathrm{n}}+\frac{1}{2 !} \mathrm{f}^{\prime \prime}(\xi) \mathrm{e}_{\mathrm{n}}^{2}+\frac{1}{3 !} \mathrm{f}^{\prime \prime \prime}(\xi) \mathrm{e}_{\mathrm{n}}^{3}+\frac{1}{4 !} \mathrm{f}^{(4)} \mathrm{e}_{\mathrm{n}}^{4}+ \\
& \frac{1}{5 !} \mathrm{f}^{(5)} \mathrm{e}_{\mathrm{n}}^{5}+\frac{1}{6 !} \mathrm{f}^{(6)}(\xi) \mathrm{e}_{\mathrm{n}}^{6}+\mathrm{O}\left(\mathrm{e}_{\mathrm{n}}^{7}\right) \\
= & \mathrm{f}^{\prime}(\xi)\left[\mathrm{e}_{\mathrm{n}}+\mathrm{C}_{2} \mathrm{e}_{\mathrm{n}}^{2}+\mathrm{C}_{3} \mathrm{e}_{\mathrm{n}}^{3}+\mathrm{C}_{4} \mathrm{e}_{\mathrm{n}}^{4}+\mathrm{C}_{5} \mathrm{e}_{\mathrm{n}}^{5}+\mathrm{C}_{6} \mathrm{e}_{\mathrm{n}}^{6}+\mathrm{O}\left(\mathrm{e}_{\mathrm{n}}^{7}\right)\right]
\end{aligned}
$$

where $C_{i}=\frac{1}{i !} \frac{f^{(i)}(\xi)}{f^{\prime}(\xi)}$.

furthermore, we have

$\mathrm{f}\left({ }^{\prime} \mathrm{x}_{\mathrm{n}}\right)=\mathrm{f}^{\prime}(\xi)\left[1+2 \mathrm{C}_{2} \mathrm{e}_{\mathrm{n}}+3 \mathrm{C}_{3} \mathrm{e}_{\mathrm{n}}^{2}+4 \mathrm{C}_{4} \mathrm{e}_{\mathrm{n}}^{3}+5 \mathrm{C}_{5} \mathrm{e}_{\mathrm{n}}^{4}+6 \mathrm{C}_{6} \mathrm{e}_{\mathrm{n}}^{5}+7 \mathrm{C}_{7} \mathrm{e}_{\mathrm{n}}^{6}+\mathrm{O}\left(\mathrm{e}_{\mathrm{n}}^{7}\right)\right]$

From (11), (13) and (14) we have 


$$
\begin{aligned}
x_{n+1}= & z_{n}-\frac{f\left(z_{n}\right)}{f^{\prime}\left(z_{n}\right)} \\
= & z_{n}-\left[\left(z_{n}-\xi\right)+\left(z_{n}-\xi\right)^{2} C_{2}+\left(z_{1}-\xi\right)^{3} C_{3}+O\left(e_{n}^{10}\right)\right] \times \\
& {\left[1+2\left(z_{n}-\xi\right) C_{2}+3\left(z_{n}-\xi\right)^{2} C_{3}+O\left(e_{n}^{9}\right)\right]^{-1} } \\
= & z_{n}-\left[\left(z_{n}-\xi\right)+\left(z_{n}-\xi\right)^{2} C_{2}+\left(z_{n}-\xi\right)^{3} C_{3}+O\left(e_{n}^{10}\right)\right] \times \\
= & {\left[1-2\left(z_{n}-\xi\right) C_{2}+\left(4 C_{2}^{2}-3 C_{3}\right)\left(z_{n}-\xi\right)^{2}+O\left(e_{n}^{9}\right)\right] } \\
& {\left[\left(z_{n}-\xi\right)-\left(z_{n}-\xi\right)^{2} C_{2}+O\left(e_{n}^{9}\right)\right] }
\end{aligned}
$$

Thus,

$$
\begin{aligned}
\mathrm{e}_{\mathrm{n}+1} & =\left(\mathrm{z}_{\mathrm{n}}-\xi\right)^{2} \mathrm{C}_{2}+\mathrm{O}\left(\mathrm{e}_{\mathrm{n}}^{9}\right) \\
& =\mathrm{C}_{2}^{3} \mathrm{C}_{3}^{2} \mathrm{e}_{\mathrm{n}}^{8}+\mathrm{O}\left(\mathrm{e}_{\mathrm{n}}^{9}\right) .
\end{aligned}
$$

Equation (15) illustrates that the modified Newton's methods (11) have eighth-order convergence. Now, again dividing (13) by (14)

$$
\begin{aligned}
\frac{f\left(x_{n}\right)}{f\left(x_{n}\right)}= & e_{n}-C_{2} e_{n}^{2}+\left(2 C_{2}^{2}-2 C_{3}\right) e_{n}^{3}+\left(-4 C_{2}^{3}+7 C_{2} C_{3}-3 C_{4}\right) e_{n}^{4}+ \\
& \left(8 C_{2}^{4}-20 C_{2}^{2} C_{3}-6 C_{3}^{2}+10 C_{2} C_{4}-4 C_{5}\right) e_{n}^{5}+ \\
& \left(-16 C_{2}^{5}+52 C_{2}^{3} C_{3}-28 C_{2}^{2} C_{4}+17 C_{3} C_{4}-33 C_{2} C_{3}^{2}+13 C_{2} C_{5}-5 C_{6}\right) e_{n}^{5}+O\left(e_{n}^{7}\right)
\end{aligned}
$$

using (16) we obtain

$$
\begin{aligned}
& y_{n}= x_{n}-\frac{f\left(x_{n}\right)}{f\left({ }^{\prime} x_{n}\right)} \\
&= \xi+e_{n}-\left[\begin{array}{l}
e_{n}-C_{2} e_{n}^{2}+\left(2 C_{2}^{2}-2 C_{3}\right) e_{n}^{3}+\left(-4 C_{2}^{3}+7 C_{2} C_{3}-3 C_{4}\right) e_{n}^{4}+ \\
\left(8 C_{2}^{4}-20 C_{2}^{2} C_{3}-6 C_{3}^{2}+10 C_{2} C_{4}-4 C_{5}\right) e_{n}^{5}+ \\
\left.\left(-16 C_{2}^{5}+52 C_{2}^{3} C_{3}-28 C_{2}^{2} C_{4}+17 C_{3} C_{4}-33 C_{2} C_{3}^{2}+13 C_{2} C_{5}-5 C_{6}\right) e_{n}^{5}+O\left(e_{n}^{7}\right)\right]
\end{array}\right. \\
&=\xi+C_{2} e_{n}^{2}+\left(2 C_{3}-2 C_{2}^{2}\right) e_{n}^{3}+\left(4 C_{2}^{3}-7 C_{2} C_{3}+3 C_{4}\right) e_{n}^{4}+ \\
&\left(-8 C_{2}^{4}+20 C_{2}^{2} C_{3}-6 C_{3}^{2}-10 C_{2} C_{4}+4 C_{5}\right) e_{n}^{5}+ \\
&\left(16 C_{2}^{5}-52 C_{2}^{3} C_{3}+28 C_{2}^{2} C_{4}-17 C_{3} C_{4}+33 C_{2} C_{3}^{2}-13 C_{2} C_{5}+5 C_{6}\right) e_{n}^{5}+O\left(e_{n}^{7}\right)
\end{aligned}
$$

by considering (17) we have the Taylor's expansion of

$$
f\left(y_{n}\right)=f^{\prime}(\xi)\left[\begin{array}{l}
C_{2} e_{n}^{2}+\left(2 C_{3}-2 C_{2}^{2}\right) e_{n}^{3}+\left(5 C_{2}^{3}-7 C_{2} C_{3}+3 C_{4}\right) e_{n}^{4}+ \\
\left(-12 C_{2}^{4}+24 C_{2}^{2} C_{3}-6 C_{3}^{2}-10 C_{2} C_{4}+4 C_{5}\right) e_{n}^{5}+ \\
\left(28 C_{2}^{5}-73 C_{2}^{3} C_{3}+34 C_{2}^{2} C_{4}-17 C_{3} C_{4}+37 C_{2} C_{3}^{2}-13 C_{2} C_{5}+5 C_{6}\right) e_{n}^{5}+ \\
O\left(e_{n}^{7}\right)
\end{array}\right]
$$

Using (13), (14) and (18) we obtain 


$$
\begin{aligned}
\mathrm{z}_{\mathrm{n}} & =\mathrm{y}_{\mathrm{n}}-\left[\frac{2 \mathrm{f}\left(\mathrm{x}_{\mathrm{n}}\right)-\mathrm{f}\left(\mathrm{y}_{\mathrm{n}}\right)}{2 \mathrm{f}\left(\mathrm{x}_{\mathrm{n}}\right)-5 \mathrm{f}\left(\mathrm{y}_{\mathrm{n}}\right)}\right] \frac{\mathrm{f}\left(\mathrm{y}_{\mathrm{n}}\right)}{\mathrm{f}^{\prime}\left(\mathrm{x}_{\mathrm{n}}\right)} \\
& =\xi-\mathrm{C}_{2} \mathrm{C}_{3} \mathrm{e}_{\mathrm{n}}^{4}+\left(-2 \mathrm{C}_{2} \mathrm{C}_{4}+2 \mathrm{C}_{2}^{2} \mathrm{C}_{3}+\frac{3}{2} \mathrm{C}_{2}^{4}-2 \mathrm{C}_{3}^{2}\right) \mathrm{e}_{\mathrm{n}}^{5} \\
& +\left(-12 \mathrm{C}_{2} \mathrm{C}_{5}-7 \mathrm{C}_{3} \mathrm{C}_{4}+3 \mathrm{C}_{2}^{2} \mathrm{C}_{4}+6 \mathrm{C}_{3} \mathrm{C}_{2}^{3}-\frac{35}{4} \mathrm{C}_{2}^{5}\right) \mathrm{e}_{\mathrm{n}}^{6}+O\left(\mathrm{e}_{\mathrm{n}}^{6}\right)
\end{aligned}
$$

by considering (19) we have the Taylor's expansion of

$$
f\left(z_{n}\right)=f(\xi)\left[\begin{array}{l}
-C_{2} C_{3} e_{n}^{4}+\left(-2 C_{2} C_{4}+2 C_{2}^{2} C_{3}+\frac{3}{2} C_{2}^{4}-2 C_{3}^{2}\right) e_{n}^{5}+ \\
\left(-12 C_{2} C_{5}-7 C_{3} C_{4}+3 C_{2}^{2} C_{4}+6 C_{3} C_{2}^{3}-\frac{35}{4} C_{2}^{5}\right) e_{n}^{6}+ \\
O\left(e_{n}^{7}\right)
\end{array}\right]
$$

now, form formulas (13),(17),(18),(19), and (20) we have

$$
\left(z_{n}-x_{n}\right)^{2} f\left(y_{n}\right)=\left[\begin{array}{l}
C_{2} e_{n}^{4}+\left(2 C_{3}-2 C_{2}^{2}\right) e_{n}^{5}+\left(5 C_{2}^{3}-7 C_{2} C_{3}+3 C_{4}\right) e_{n}^{6}+ \\
+\left(-12 C_{2}^{4}+26 C_{3} C_{2}^{2}-6 C_{3}^{2}-10 C_{2} C_{4}+4 C_{5}\right) e_{n}^{7}+ \\
{\left[\begin{array}{l}
25 C_{2}^{5}-81 C_{3} C_{2}^{3}+38 C_{4} C_{2}^{2}-17 C_{3} C_{4}+ \\
45 C_{2} C_{3}^{2}-13 C_{2} C_{5}+5 C_{6}
\end{array}\right] e_{n}^{8}+O\left(e_{n}^{9}\right)}
\end{array}\right]
$$$$
\left(y_{n}-x_{n}\right)\left(y_{n}-z_{n}\right) f\left(z_{n}\right)=C_{2}^{2} C_{3} e_{n}^{7}+\left(2 C_{4} C_{2}^{2}+4 C_{2} C_{3}^{2}-5 C_{3} C_{2}^{3}-\frac{3}{2} C_{2}^{5}\right) e_{n}^{8}+O\left(e_{n}^{9}\right)
$$$$
\left(y_{n}-z_{n}\right)^{2} f\left(x_{n}\right)=\left[\begin{array}{l}
C_{2}^{2} e_{n}^{5}+\left(-3 C_{2}^{3}+4 C_{2} C_{3}\right) e_{n}^{6} \\
+\left(8 C_{2}^{4}-15 C_{3} C_{2}^{2}+4 C_{3}^{2}+6 C_{2} C_{4}\right) e_{n}^{7}+ \\
{\left[\begin{array}{l}
-23 C_{2}^{5}+52 C_{3} C_{2}^{3}-11 C_{4} C_{2}^{2}-12 C_{3} C_{4}- \\
24 C_{2} C_{3}^{2}+8 C_{2} C_{5}
\end{array}\right] e_{n}^{8}+O\left(e_{n}^{9}\right)}
\end{array}\right]
$$

$$
\left(y_{n}-x_{n}\right)\left(z_{n}-x_{n}\right) f\left(z_{n}\right)=\left[\begin{array}{l}
-C_{2} C_{3} e_{n}^{6}+\left(\frac{3}{2} C_{2}^{4}+3 C_{3} C_{2}^{2}-2 C_{3}^{2}-2 C_{2} C_{4}\right) e_{n}^{7}+ \\
{\left[\begin{array}{l}
\frac{41}{4} C_{2}^{5}+2 C_{3} C_{2}^{3}+5 C_{4} C_{2}^{2}-7 C_{3} C_{4}+ \\
45 C_{2} C_{3}^{2}-3 C_{2} C_{5}
\end{array}\right] e_{n}^{8}+O\left(e_{n}^{9}\right)}
\end{array}\right]
$$

$\Rightarrow$

$$
\left[\begin{array}{l}
\left(z_{n}-x_{n}\right)^{2} f\left(y_{n}\right)+ \\
\left(y_{n}-x_{n}\right)\left(y_{n}-z_{n}\right) f\left(z_{n}\right)- \\
\left(y_{n}-z_{n}\right)^{2} f\left(x_{n}\right)- \\
\left(y_{n}-x_{n}\right)\left(z_{n}-x_{n}\right) f\left(z_{n}\right)
\end{array}\right]=C_{2} e_{n}^{4}+\left(2 C_{3}-3 C_{2}^{2}\right) e_{n}^{5}+\left(8 C_{2}^{3}-10 C_{2} C_{3}+3 C_{4}\right) e_{n}^{6}+
$$

$$
\left(-\frac{43}{2} C_{2}^{4}+39 C_{3} C_{2}^{2}-8 C_{3}^{2}-14 C_{2} C_{4}+4 C_{5}\right) e_{n}^{7}+O\left(e_{n}^{8}\right)
$$


And

$$
\begin{aligned}
\left(y_{n}-x_{n}\right)\left(z_{n}-x_{n}\right)\left(y_{n}-z_{n}\right)= & C_{2} e_{n}^{4}+\left(2 C_{3}-3 C_{2}^{2}\right) e_{n}^{5}+\left(8 C_{2}^{3}-10 C_{2} C_{3}+3 C_{4}\right) e_{n}^{6}+ \\
& \left(-\frac{43}{2} C_{2}^{4}+40 C_{3} C_{2}^{2}-8 C_{3}^{2}-14 C_{2} C_{4}+4 C_{5}\right) e_{n}^{7}+O\left(e_{n}^{8}\right)
\end{aligned}
$$

from equations (20), (21) and (22)

$$
\begin{aligned}
x_{n+1}= & z_{n}-\frac{\left(y_{n}-x_{n}\right)\left(y_{n}-z_{n}\right)\left(z_{n}-x_{n}\right) f\left(z_{n}\right)}{\left[\left(z_{n}-x_{n}\right)^{2} f\left(y_{n}\right)+\left(y_{n}-x_{n}\right)\left(y_{n}-z_{n}\right) f\left(z_{n}\right)-\left(y_{n}-z_{n}\right)^{2} f\left(x_{n}\right)-\left(y_{n}-x_{n}\right)\left(z_{n}-x_{n}\right) f\left(z_{n}\right)\right]} \\
& =z_{n}-\left[\left(z_{n}-\xi\right)+\left(z_{n}-\xi\right)^{2} C_{2}+\left(z_{n}-\xi\right)^{3} C_{3}+O\left(e_{n}^{16}\right)\right] \times\left[1+C_{3} C_{2}^{2} e_{n}^{3}+O\left(e_{n}^{4}\right)\right] \\
& =z_{n}-\left[\left(z_{n}-\xi\right)+C_{3} C_{2}\left(z_{n}-\xi\right) e_{n}^{3}+O\left(e_{n}^{8}\right)\right]
\end{aligned}
$$

Thus,

$$
\begin{aligned}
\mathrm{e}_{\mathrm{n}+1} & =\mathrm{C}_{3} \mathrm{C}_{2}^{2}\left(\mathrm{z}_{\mathrm{n}+1}-\xi\right) \mathrm{e}_{\mathrm{n}}^{2}+\mathrm{O}\left(\mathrm{e}_{\mathrm{n}}^{8}\right) \\
& =-\mathrm{C}_{2}^{2} \mathrm{C}_{3}^{2} \omega \mathrm{e}_{\mathrm{n}}^{7}+\mathrm{O}\left(\mathrm{e}_{\mathrm{n}}^{8}\right)
\end{aligned}
$$

Equation (23) illustrates that the modified Newton's methods (12) have seventh-order convergence.

Now we develop second modification of the method defined by (9), (11) and (12) to restore order of convergence eight.

Let us take the following parameter

$$
\left\{\begin{array}{l}
x_{n+1}=z_{n}-\frac{\left(y_{n}-x_{n}\right)\left(y_{n}-z_{n}\right)\left(z_{n}-x_{n}\right) f\left(z_{n}\right)}{\left[\begin{array}{l}
\left(z_{n}-x_{n}\right)^{2} f\left(y_{n}\right)+\beta\left(y_{n}-x_{n}\right)\left(y_{n}-z_{n}\right) f\left(z_{n}\right)- \\
\left(y_{n}-z_{n}\right)^{2} f\left(x_{n}\right)-\left(y_{n}-x_{n}\right)\left(z_{n}-x_{n}\right) f\left(z_{n}\right)
\end{array}\right]} \\
z_{n}=y_{n}-\left[\frac{2 f\left(x_{n}\right)-f\left(y_{n}\right)}{2 f\left(x_{n}\right)-5 f\left(y_{n}\right)}\right] \frac{f\left(y_{n}\right)}{f^{\prime}\left(x_{n}\right)} \\
y_{n}=x_{n}-\frac{f\left(x_{n}\right)}{f^{\prime}\left(x_{n}\right)}
\end{array}\right.
$$

Here, $b$ is yet to be determined. Now, it will be shown that, for appropriate value of parameter, the method defined by (9), (11) and (12) converges with order 8 in the vicinity of root.

Again by (21) and (22)

$$
\begin{aligned}
\mathrm{x}_{\mathrm{n}+1} & =\mathrm{z}_{\mathrm{n}}-\frac{\left(\mathrm{y}_{\mathrm{n}}-\mathrm{x}_{\mathrm{n}}\right)\left(\mathrm{y}_{\mathrm{n}}-\mathrm{z}_{\mathrm{n}}\right)\left(\mathrm{z}_{\mathrm{n}}-\mathrm{x}_{\mathrm{n}}\right) \mathrm{f}\left(\mathrm{z}_{\mathrm{n}}\right)}{\left[\left(\mathrm{z}_{\mathrm{n}}-\mathrm{x}_{\mathrm{n}}\right)^{2} \mathrm{f}\left(\mathrm{y}_{\mathrm{n}}\right)+\left(\mathrm{y}_{\mathrm{n}}-\mathrm{x}_{\mathrm{n}}\right)\left(\mathrm{y}_{\mathrm{n}}-\mathrm{z}_{\mathrm{n}}\right) \mathrm{f}\left(\mathrm{z}_{\mathrm{n}}\right)-\left(\mathrm{y}_{\mathrm{n}}-\mathrm{z}_{\mathrm{n}}\right)^{2} \mathrm{f}\left(\mathrm{x}_{\mathrm{n}}\right)-\left(\mathrm{y}_{\mathrm{n}}-\mathrm{x}_{\mathrm{n}}\right)\left(\mathrm{z}_{\mathrm{n}}-\mathrm{x}_{\mathrm{n}}\right) \mathrm{f}\left(\mathrm{z}_{\mathrm{n}}\right)\right]} \\
& =\xi+(2-\beta) \mathrm{C}_{3} \mathrm{C}_{2}^{2} \mathrm{e}_{\mathrm{n}}^{7}+\left[\begin{array}{l}
(77-3 \beta) \mathrm{C}_{2}^{5} \mathrm{C}_{3}+(10-7 \beta) \mathrm{C}_{3}^{2} \mathrm{C}_{2}^{3}+(4 \beta-17) \mathrm{C}_{3} \mathrm{C}_{4} \mathrm{C}_{2}^{2}+ \\
(6 \beta-12) \mathrm{C}_{2} \mathrm{C}_{3}^{2}+24 \mathrm{C}_{4} \mathrm{C}_{3}^{2}
\end{array}\right] \mathrm{e}_{\mathrm{n}}^{8}+\mathrm{O}\left(\mathrm{e}_{\mathrm{n}}^{9}\right)
\end{aligned}
$$

If we choose $\beta=2$, we have the eighth order method, with the error at becomes 


$$
e_{n+1}=\left[71 C_{2}^{5} C_{3}+-4 C_{3}^{2} C_{2}^{3}-9 C_{3} C_{4} C_{2}^{2}+24 C_{4} C_{3}^{2}\right] e_{n}^{8}+O\left(e_{n}^{9}\right)
$$

Now for generating a new modification of the Newton's method with seventh -order convergence, we suggest the following scheme:

$$
\left\{\begin{array}{l}
x_{n+1}=z_{n}-\frac{\left(y_{n}-x_{n}\right)\left(y_{n}-z_{n}\right)\left(z_{n}-x_{n}\right) f\left(z_{n}\right)}{\left[\begin{array}{l}
\left(z_{n}-x_{n}\right)^{2} f\left(y_{n}\right)+\beta\left(y_{n}-x_{n}\right)\left(y_{n}-z_{n}\right) f\left(z_{n}\right)- \\
\left(y_{n}-z_{n}\right)^{2} f\left(x_{n}\right)-\left(y_{n}-x_{n}\right)\left(z_{n}-x_{n}\right) f\left(z_{n}\right)
\end{array}\right]} \\
z_{n}=y_{n}-\left[\frac{2 f\left(x_{n}\right)-f\left(y_{n}\right)}{2 f\left(x_{n}\right)-5 f\left(y_{n}\right)}\right] \frac{f\left(y_{n}\right)}{f^{\prime}\left(x_{n}\right)}, \\
y_{n}=x_{n}-\frac{f\left(x_{n}\right)}{f^{\prime}\left(x_{n}\right)}
\end{array},\right.
$$

Also for the modified Newton's method with eighth-order convergence, we suggest the following iterative scheme :

$$
\left\{\begin{array}{l}
x_{n+1}=z_{n}-\frac{\left(y_{n}-x_{n}\right)\left(y_{n}-z_{n}\right)\left(z_{n}-x_{n}\right) f\left(z_{n}\right)}{\left[\begin{array}{l}
\left.\left(z_{n}-x_{n}\right)^{2} f\left(y_{n}\right)+2\left(y_{n}-x_{n}\right)\left(y_{n}-z_{n}\right) f\left(z_{n}\right)-\right] \\
\left(y_{n}-z_{n}\right)^{2} f\left(x_{n}\right)-\left(y_{n}-x_{n}\right)\left(z_{n}-x_{n}\right) f\left(z_{n}\right)
\end{array}\right]} \\
z_{n}=y_{n}-\left[\frac{2 f\left(x_{n}\right)-f\left(y_{n}\right)}{2 f\left(x_{n}\right)-5 f\left(y_{n}\right)}\right] \frac{f\left(y_{n}\right)}{f^{\prime}\left(x_{n}\right)}, \\
y_{n}=x_{n}-\frac{f\left(x_{n}\right)}{f^{\prime}\left(x_{n}\right)}
\end{array},\right.
$$

\section{Numerical examples}

In this section, we consider some numerical examples to demonstrate the performance and the efficiency of the modified Newton's methods with ( seventh or eighth )-order convergence. All computations were done by $C^{++}$.

The following test functions have been used with termination criterion $\left|f\left(x_{n}\right)\right|<10^{-15}$. 
Table 1. Results of the Modified Newton's Methods with Seventh Or Eighth Order Convergence.

\begin{tabular}{|c|c|c|c|c|c|c|c|c|c|c|}
\hline \multirow{2}{*}{$f(x)$} & \multirow{2}{*}{$x_{0}$} & \multicolumn{2}{|c|}{ C-NM } & \multicolumn{2}{|c|}{$\mathrm{mNm}(9)$} & \multicolumn{2}{|c|}{$\mathrm{mNm}(24)$} & \multicolumn{2}{|c|}{$\mathrm{mNm}(25)$} & \multirow{2}{*}{$\frac{\text { Root }}{\xi}$} \\
\hline & & $\mathrm{n}$ & $\mathrm{m}$ & $\mathrm{n}$ & $\mathrm{m}$ & $\mathrm{n}$ & $\mathrm{m}$ & $\mathrm{n}$ & $\mathrm{m}$ & \\
\hline \multirow{4}{*}{$x^{3}+4 x^{2}-10$} & -0.3 & 54 & 108 & 6 & 18 & 18 & 54 & 5 & 15 & \multirow[t]{4}{*}{1.36523001341410} \\
\hline & -0.25 & 25 & 50 & 8 & 24 & 6 & 18 & 5 & 15 & \\
\hline & 0.25 & 8 & 16 & 6 & 18 & 5 & 15 & 5 & 15 & \\
\hline & 1 & 5 & 10 & 3 & 9 & 3 & 9 & 3 & 9 & \\
\hline \multirow{3}{*}{$x^{11}+x^{6}+x-1$} & -3 & 36 & 72 & 15 & 45 & 22 & 66 & 15 & 45 & \multirow[t]{3}{*}{0.759516263131002} \\
\hline & 2 & 13 & 26 & 9 & 27 & 8 & 24 & 9 & 27 & \\
\hline & 3 & 18 & 36 & 12 & 36 & 11 & 33 & 11 & 33 & \\
\hline \multirow[t]{6}{*}{$\cos (x)-x$} & -10 & 21 & 42 & 6 & 18 & 6 & 18 & 5 & 15 & \multirow[t]{6}{*}{0.739085133215161} \\
\hline & -3 & 24 & 48 & 4 & 12 & 9 & 27 & 5 & 15 & \\
\hline & -2 & 7 & 14 & 4 & 12 & 5 & 15 & 5 & 15 & \\
\hline & -1.5 & 38 & 76 & 7 & 21 & 8 & 24 & 4 & 12 & \\
\hline & 0 & 5 & 10 & 3 & 9 & 3 & 9 & 3 & 9 & \\
\hline & 10 & 70 & 14 & 9 & 27 & 9 & 27 & 5 & 15 & \\
\hline \multirow{2}{*}{$(x+1)^{2} e^{\left(x^{2}-2\right)}-1$} & 0.75 & 5 & 10 & 3 & 9 & 3 & 9 & 3 & 9 & \multirow[t]{2}{*}{0.866873543487685} \\
\hline & 1 & 5 & 10 & 3 & 9 & 3 & 9 & 3 & 9 & \\
\hline \multirow[t]{3}{*}{$(x-1)^{3}-1$} & -10 & 19 & 38 & 8 & 24 & 12 & 36 & 8 & 24 & \multirow[t]{3}{*}{2} \\
\hline & 0.25 & 16 & 32 & 4 & 12 & 6 & 18 & 5 & 15 & \\
\hline & 2.5 & 6 & 12 & 4 & 12 & 4 & 12 & 4 & 12 & \\
\hline \multirow{2}{*}{$\begin{array}{l}x e^{x^{2}}-\sin ^{2}(x)+ \\
3 \cos (x)+5\end{array}$} & -3 & 15 & 30 & 9 & 27 & 9 & 27 & 9 & 27 & \multirow[t]{2}{*}{-1.207647827130} \\
\hline & -1 & 6 & 12 & 4 & 12 & 4 & 12 & 4 & 12 & \\
\hline
\end{tabular}

$\mathrm{n} /$ Number of iterations to approximate the root to 15 decimal places.

$\mathrm{m} /$ Number of function evaluations.

C -NM/ The classical Newton's method.

\section{Conclusions}

In the table .1 we observe that the modified Newton's method with (seventh or eighth)-order of convergence satisfied great successful through their application for finding a simple real roots of non-linear equations,

comparing with all the methods cited in the table .1. Therefore by considering the efficiency index $r^{\frac{1}{n}}$ where $r$ is the order of convergence of the method and $n$ is the number of function evaluations required by the method (units of work for iteration), in the $\mathrm{mNm}$ (24) and $\mathrm{mNm}$ (25) we have efficiency index equal $\sqrt[4]{7} \cong 1.6265766$ and $\sqrt[4]{8} \cong 1.681793$ respectively, which are better than efficiency index of modified Newton's methods with the fourth-order of convergence $\sqrt[3]{4} \cong 1.587410$, and efficiency index of classical Newton's method $\sqrt{2} \cong 1.414$. However, In some applications that require a number of repeated tests, the increasing of order may draw a concrete advantage if the cost of computational complexity, implied by the increased order, is justified from the advantage in terms of execution time. 


\section{References}

[1] H.H.H. Homeier, On Newton's -type methods with cubic convergence, J. comput. Appl. Math. 176 (2005) 425-432.

[2] J. F. Traub, Iterative methods for the solution of equations, Chelsea Publishing Company, New York, 1977.

[3] M. Frontini, E. Sormani, Some variants of Newton's method with third -order convergence, Appl. Math. Copmut . 140(2003),pp.419- 426.

[4] R. F. Kung, A falimly of fourth order methods for nonlinear equtions, SIAM Journal on Numerical Analysis. 10 (1973), pp.876-879.

[5] R. L. Burden, J. D. Faires, Numerical analysis, PWS Publishing Company, Fifth edition, USA, 1993.

[6] S. Weerakoom, T.G.I. Fernando, A variant of Newton's method with accelerated thirdorder convergence, Appl. Math . Lett .13 (2000), pp.87-93. 